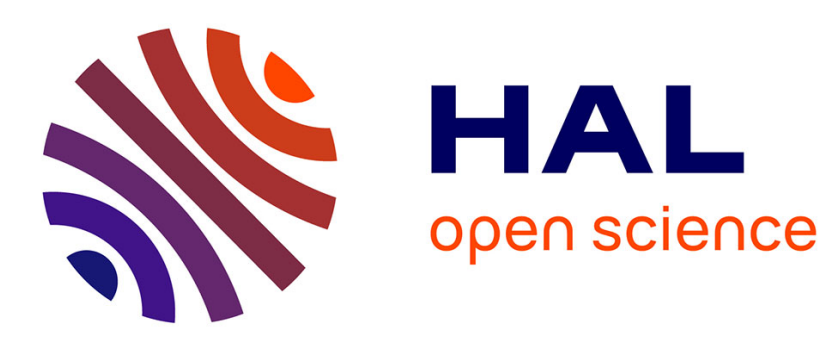

\title{
Stochastic modeling of the oceanic mesoscale eddies
}

\author{
Long Li, Etienne Mémin, Deremble Bruno
}

\section{To cite this version:}

Long Li, Etienne Mémin, Deremble Bruno. Stochastic modeling of the oceanic mesoscale eddies. Stochastic Transport in Upper Ocean Dynamics Workshop, Sep 2020, London, United Kingdom. hal02946702

\section{HAL Id: hal-02946702 \\ https://hal.science/hal-02946702}

Submitted on 23 Sep 2020

HAL is a multi-disciplinary open access archive for the deposit and dissemination of scientific research documents, whether they are published or not. The documents may come from teaching and research institutions in France or abroad, or from public or private research centers.
L'archive ouverte pluridisciplinaire HAL, est destinée au dépôt et à la diffusion de documents scientifiques de niveau recherche, publiés ou non, émanant des établissements d'enseignement et de recherche français ou étrangers, des laboratoires publics ou privés. 


\title{
Stochastic modeling of the oceanic mesoscale eddies
}

\author{
Long $\mathrm{Li}^{1} \quad$ Etienne Mémin ${ }^{1} \quad$ Deremble Bruno ${ }^{2}$
}

${ }^{1}$ FLUMINANCE Group

INRIA Rennes, France

${ }^{2}$ MEOM Group

IGE Grenoble, France 


\section{Motivations}

- To better represent the mesoscale eddies effect on the large-scale circulations. 


\section{Motivations}

- To better represent the mesoscale eddies effect on the large-scale circulations.

- To improve the prediction of ocean variability in very coarse-grid simulations. 


\section{Motivations}

- To better represent the mesoscale eddies effect on the large-scale circulations.

- To improve the prediction of ocean variability in very coarse-grid simulations.

- To provide a more reliable ensemble forecasting system and more efficient ensemble spread for data assimilation system. 


\section{Outline}

(1) Location Uncertainty (LU) model 


\section{LU model}

Stochastic flow (Mémin, 2014)

$$
\mathrm{d} \boldsymbol{X}_{t}=\boldsymbol{u}\left(\boldsymbol{X}_{t}, t\right) \mathrm{d} t+\underbrace{\boldsymbol{\sigma}\left(\boldsymbol{X}_{t}, t\right) \mathrm{d} \boldsymbol{B}_{t}}_{\text {Uncertainty or Noise }}
$$




\section{LU model}

\section{Spatial structure of noise}

- Correlation operator

$$
\boldsymbol{\sigma}[\boldsymbol{f}](\boldsymbol{x}, t)=\int_{\Omega} \breve{\boldsymbol{\sigma}}(\boldsymbol{x}, \boldsymbol{y}, t) \boldsymbol{f}(\boldsymbol{y}) \mathrm{d} \boldsymbol{y}
$$

- $\breve{\sigma}$ is assumed to be bounded 


\section{LU model}

\section{Spatial structure of noise}

- Correlation operator

$$
\boldsymbol{\sigma}[\boldsymbol{f}](\boldsymbol{x}, t)=\int_{\Omega} \breve{\boldsymbol{\sigma}}(\boldsymbol{x}, \boldsymbol{y}, t) \boldsymbol{f}(\boldsymbol{y}) \mathrm{d} \boldsymbol{y}
$$

- Variance tensor

$$
\boldsymbol{a}=\mathbb{E}\left[\left(\boldsymbol{\sigma} \mathrm{d} \boldsymbol{B}_{t}\right)\left(\boldsymbol{\sigma} \mathrm{d} \boldsymbol{B}_{t}\right)^{T}\right] / \mathrm{d} t=\boldsymbol{\sigma} \boldsymbol{\sigma}^{T}
$$

- $\breve{\sigma}$ is assumed to be bounded 


\section{LU model}

\section{Spectral representation}

- Noise

$$
\boldsymbol{\sigma}(\boldsymbol{x}, t) \mathrm{d} \boldsymbol{B}_{t}=\sum_{n \in \mathbb{N}} \phi_{n}(\boldsymbol{x}, t) \mathrm{d} \beta_{t}^{n}
$$

- $\phi_{n}$ : orthogonal eigenfunctions of the covariance

- $\beta^{n}:$ 1D standard Brownian motions 


\section{LU model}

\section{Spectral representation}

- Noise

$$
\boldsymbol{\sigma}(\boldsymbol{x}, t) \mathrm{d} \boldsymbol{B}_{t}=\sum_{n \in \mathbb{N}} \phi_{n}(\boldsymbol{x}, t) \mathrm{d} \beta_{t}^{n}
$$

- Variance

$$
\boldsymbol{a}(\boldsymbol{x}, t)=\sum_{n \in \mathbb{N}} \boldsymbol{\phi}_{n}(\boldsymbol{x}, t) \boldsymbol{\phi}_{n}^{T}(\boldsymbol{x}, t)
$$

- $\phi_{n}$ : orthogonal eigenfunctions of the covariance

- $\beta^{n}:$ 1D standard Brownian motions 


\section{LU model}

\section{Transport of extensive tracers (Resseguier et al., 2017)}

- Stochastic transport operator

$$
\mathbb{D}_{t} \theta \triangleq \mathrm{d}_{t} \theta+\left(\boldsymbol{u}-\boldsymbol{u}_{s}\right) \cdot \boldsymbol{\nabla} \theta \mathrm{d} t+\underbrace{\boldsymbol{\sigma} \mathrm{d} \boldsymbol{B}_{t} \cdot \boldsymbol{\nabla} \theta}_{\text {forcing }}-\underbrace{\frac{1}{2} \boldsymbol{\nabla} \cdot(\boldsymbol{a} \boldsymbol{\nabla} \theta) \mathrm{d} t}_{\text {diffusion }}=0
$$

- Incompressible noise: $\boldsymbol{\nabla} \cdot \boldsymbol{\sigma}=0$

- Itô-Stokes drift (Bauer et al., 2020a): $\boldsymbol{u}_{s} \triangleq \frac{1}{2} \boldsymbol{\nabla} \cdot \boldsymbol{a}$ 


\section{LU model}

\section{Transport of extensive tracers (Resseguier et al., 2017)}

- Stochastic transport operator

$$
\mathbb{D}_{t} \theta \triangleq \mathrm{d}_{t} \theta+\left(\boldsymbol{u}-\boldsymbol{u}_{s}\right) \cdot \boldsymbol{\nabla} \theta \mathrm{d} t+\underbrace{\boldsymbol{\sigma} \mathrm{d} \boldsymbol{B}_{t} \cdot \nabla \theta}_{\text {forcing }}-\underbrace{\frac{1}{2} \boldsymbol{\nabla} \cdot(\boldsymbol{a} \boldsymbol{\nabla} \theta) \mathrm{d} t}_{\text {diffusion }}=0
$$

- Conservation of energy

$$
\boldsymbol{\nabla} \cdot\left(\boldsymbol{u}-\boldsymbol{u}_{s}\right)=0 \quad \Longrightarrow \quad \mathrm{d}_{t} \int_{\Omega} \frac{1}{2} \theta^{2} \mathrm{~d} \boldsymbol{x}=0
$$

- Incompressible noise: $\nabla \cdot \sigma=0$

- Itô-Stokes drift (Bauer et al., 2020a): $\boldsymbol{u}_{s} \triangleq \frac{1}{2} \boldsymbol{\nabla} \cdot \boldsymbol{a}$ 


\section{LU model}

\section{Connection to physical parameterizations}

- Isopycnal projector

$$
\mathbf{P}=\mathbf{I}-\frac{\boldsymbol{\nabla} \rho(\boldsymbol{\nabla} \rho)^{T}}{|\boldsymbol{\nabla} \rho|^{2}} \quad \Longrightarrow \quad \mathbf{P} \boldsymbol{\nabla} \rho=0
$$

- Projector: $\mathbf{P}^{T}=\mathbf{P}, \quad \mathbf{P}^{2}=\mathbf{P}$ 


\section{LU model}

\section{Connection to physical parameterizations}

- Isopycnal projector

$$
\mathbf{P}=\mathbf{I}-\frac{\boldsymbol{\nabla} \rho(\boldsymbol{\nabla} \rho)^{T}}{|\boldsymbol{\nabla} \rho|^{2}} \quad \Longrightarrow \quad \mathbf{P} \boldsymbol{\nabla} \rho=0
$$

- Isopycnal noise

$$
\begin{aligned}
\boldsymbol{\sigma}=\mathbf{P} \boldsymbol{\sigma}_{0} & \Longrightarrow \quad \boldsymbol{\sigma} \mathrm{d} \boldsymbol{B}_{t} \perp \nabla \rho \text { and } \boldsymbol{a} \boldsymbol{\nabla} \rho=0 \\
& \Longrightarrow \quad \partial_{t} \rho+\nabla \cdot\left(\rho\left(\boldsymbol{u}-\boldsymbol{u}_{s}\right)\right)=0
\end{aligned}
$$

- Projector: $\mathbf{P}^{T}=\mathbf{P}, \quad \mathbf{P}^{2}=\mathbf{P}$ 


\section{LU model}

\section{Connection to physical parameterizations}

- Transport of passive tracer

$$
\mathrm{d}_{t} \theta+\left(\boldsymbol{u}-\boldsymbol{u}_{s}\right) \cdot \boldsymbol{\nabla} \theta \mathrm{d} t+\boldsymbol{\sigma} \mathrm{d} \boldsymbol{B}_{t} \cdot \boldsymbol{\nabla} \theta-\frac{1}{2} \boldsymbol{\nabla} \cdot(\boldsymbol{a} \boldsymbol{\nabla} \theta) \mathrm{d} t=0
$$




\section{LU model}

\section{Connection to physical parameterizations}

- Transport of passive tracer

$$
\mathrm{d}_{t} \theta+\left(\boldsymbol{u}-\boldsymbol{u}_{s}\right) \cdot \boldsymbol{\nabla} \theta \mathrm{d} t+\boldsymbol{\sigma} \mathrm{d} \boldsymbol{B}_{t} \cdot \boldsymbol{\nabla} \theta-\frac{1}{2} \boldsymbol{\nabla} \cdot(\boldsymbol{a} \boldsymbol{\nabla} \theta) \mathrm{d} t=0
$$

- Isopycnal diffusion (Gent and McWilliams, 1990; Redi, 1982)

$$
\boldsymbol{a}_{0}=\kappa \mathbf{I} \quad \Longrightarrow \quad \boldsymbol{a}=\mathbf{P} \boldsymbol{a}_{0} \mathbf{P}^{T}=\kappa \mathbf{P}
$$




\section{LU model}

\section{Connection to physical parameterizations}

- Transport of passive tracer

$$
\mathrm{d}_{t} \theta+\left(\boldsymbol{u}-\boldsymbol{u}_{s}\right) \cdot \boldsymbol{\nabla} \theta \mathrm{d} t+\boldsymbol{\sigma} \mathrm{d} \boldsymbol{B}_{t} \cdot \boldsymbol{\nabla} \theta-\frac{1}{2} \boldsymbol{\nabla} \cdot(\boldsymbol{a} \boldsymbol{\nabla} \theta) \mathrm{d} t=0
$$

- Isopycnal diffusion (Gent and McWilliams, 1990; Redi, 1982)

$$
\boldsymbol{a}_{0}=\kappa \mathbf{I} \quad \Longrightarrow \quad \boldsymbol{a}=\mathbf{P} \boldsymbol{a}_{0} \mathbf{P}^{T}=\kappa \mathbf{P}
$$




\section{LU model}

\section{Connection to physical parameterizations}

- Transport of passive tracer

$$
\mathrm{d}_{t} \theta+\left(\boldsymbol{u}-\boldsymbol{u}_{s}\right) \cdot \boldsymbol{\nabla} \theta \mathrm{d} t+\boldsymbol{\sigma} \mathrm{d} \boldsymbol{B}_{t} \cdot \boldsymbol{\nabla} \theta-\frac{1}{2} \boldsymbol{\nabla} \cdot(\boldsymbol{a} \boldsymbol{\nabla} \theta) \mathrm{d} t=0
$$

- Isopycnal diffusion (Gent and McWilliams, 1990; Redi, 1982)

$$
\begin{gathered}
\boldsymbol{a}_{0}=\kappa \mathbf{I} \quad \Longrightarrow \quad \boldsymbol{a}=\mathbf{P} \boldsymbol{a}_{0} \mathbf{P}^{T}=\kappa \mathbf{P} \\
|\boldsymbol{s}| \ll 1 \quad \Longrightarrow \quad \boldsymbol{a}=\kappa\left(\begin{array}{ccc}
1 & 0 & s_{x} \\
0 & 1 & s_{y} \\
s_{x} & s_{y} & |\boldsymbol{s}|^{2}
\end{array}\right)
\end{gathered}
$$

with $s=\left[s_{x}, s_{y}\right]^{T}, s_{x}=-\partial_{x} \rho / \partial_{z} \rho, s_{y}=-\partial_{y} \rho / \partial_{z} \rho$ 


\section{LU model}

\section{Quasi-geostrophic (QG) flow under LU}

- Potential vorticity (PV)

$$
q=\nabla^{2} \psi+f+f_{0}^{2} \partial_{z}\left(\partial_{z} \psi / N^{2}\right)
$$

- $\psi$ is stream function $\left(\boldsymbol{u}=\nabla^{\perp} \psi\right), f$ is Coriolis and $N$ is buoyancy frequency 


\section{LU model}

\section{Quasi-geostrophic (QG) flow under LU}

- Potential vorticity (PV)

$$
q=\nabla^{2} \psi+f+f_{0}^{2} \partial_{z}\left(\partial_{z} \psi / N^{2}\right)
$$

- Evolution of PV under LU

$$
\begin{gathered}
\mathbb{D}_{t} q=\boldsymbol{\nabla} \cdot \boldsymbol{F} \\
\boldsymbol{F} \triangleq \underbrace{-\boldsymbol{u} \cdot \boldsymbol{\nabla}^{\perp}\left(\boldsymbol{\sigma} \mathrm{d} \boldsymbol{B}_{t}-\boldsymbol{u}_{s} \mathrm{~d} t\right)-\boldsymbol{a} \boldsymbol{\nabla} f \mathrm{~d} t}_{\text {flux of PV source }} \underbrace{+\frac{1}{2}\left(\partial_{x} \boldsymbol{a} \boldsymbol{\nabla} v-\partial_{y} \boldsymbol{a} \boldsymbol{\nabla} u\right) \mathrm{d} t}_{\text {flux of PV sink }}
\end{gathered}
$$

$\boldsymbol{F}$ ensures conservation of total energy (Bauer et al., 2020a)

- $\psi$ is stream function $\left(\boldsymbol{u}=\nabla^{\perp} \psi\right), f$ is Coriolis and $N$ is buoyancy frequency 


\section{Outline}

\section{(1) Location Uncertainty (LU) model}

(2) Numerical results: LU coarse-grid simulations 


\section{Eddy-resolving simulations (Hogg et al., 2003)}

Exp.1: $L_{d}=[30,17] \mathrm{km}, \Delta=5 \mathrm{~km}$

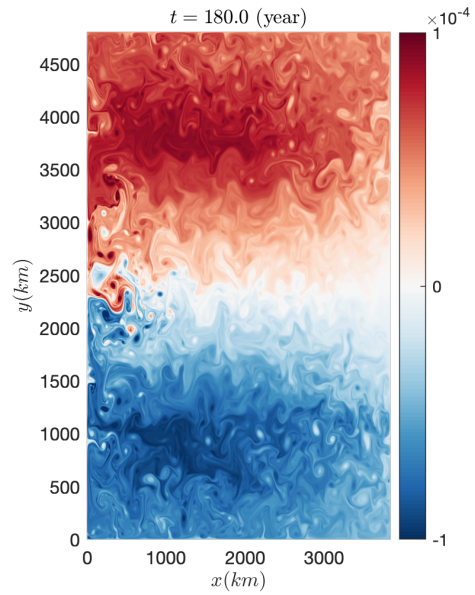

Exp.2: $L_{d}=[51,32] \mathrm{km}, \Delta=10 \mathrm{~km}$

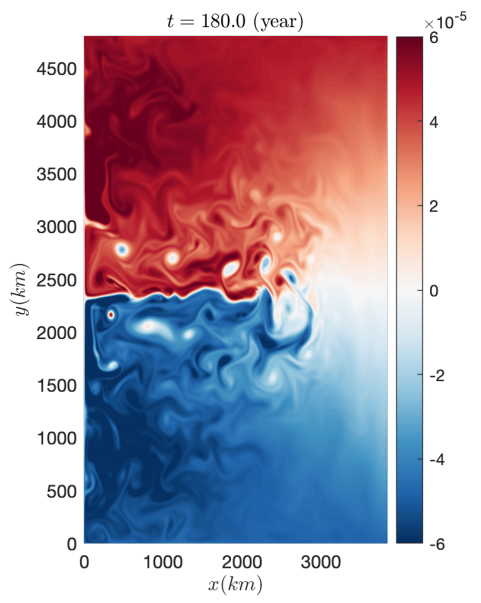

Figure: Eddy-resolving snapshots of surface layer PV (http://www.q-gcm.org). 


\section{LU-POD noise}

Learning of noise from velocity data (Bauer et al., 2020b):

$$
\frac{1}{\Delta t} \boldsymbol{\sigma}(\boldsymbol{x}) \mathrm{d} \boldsymbol{B}_{t} \approx \sum_{n=M_{0}}^{M_{1}} \boldsymbol{\phi}_{n}(\boldsymbol{x}) \xi_{n}, \quad \xi_{n} \sim \mathcal{N}(0,1)
$$
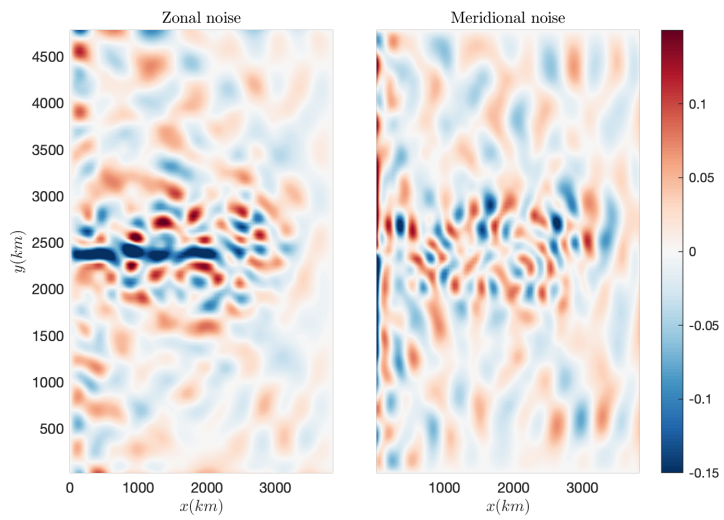

Figure: Zonal (left) and meridional (right) noise velocity at resolution $80 \mathrm{~km}$ learned from eddy-resolving data of Exp.2. 


\section{LU-POD-P noise}

Update of noise along isopycnal:

$$
\mathbf{P}=\mathbf{I}-\frac{\boldsymbol{\nabla} \eta(\boldsymbol{\nabla} \eta)^{T}}{|\boldsymbol{\nabla} \eta|^{2}}, \quad \eta \propto \partial_{z}\left(\partial_{z} \psi / N^{2}\right)
$$
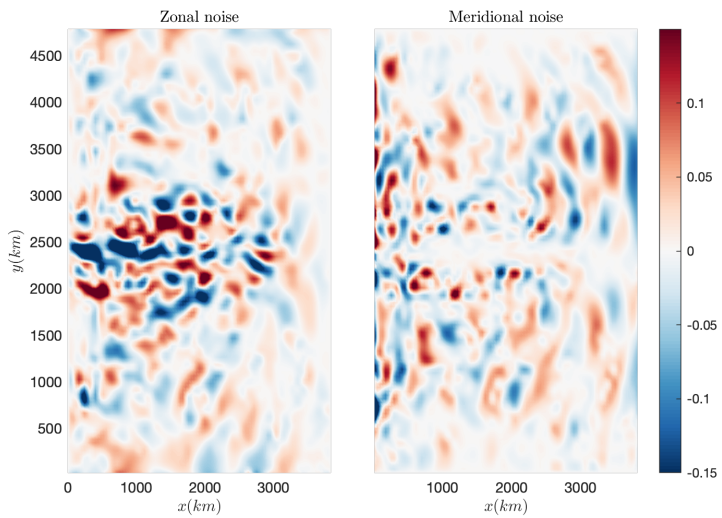

Figure: Zonal (left) and meridional (right) noise velocity at resolution $80 \mathrm{~km}$ by adapting POD noise along isopycnal. 


\section{LU coarse simulation (Exp.1)}

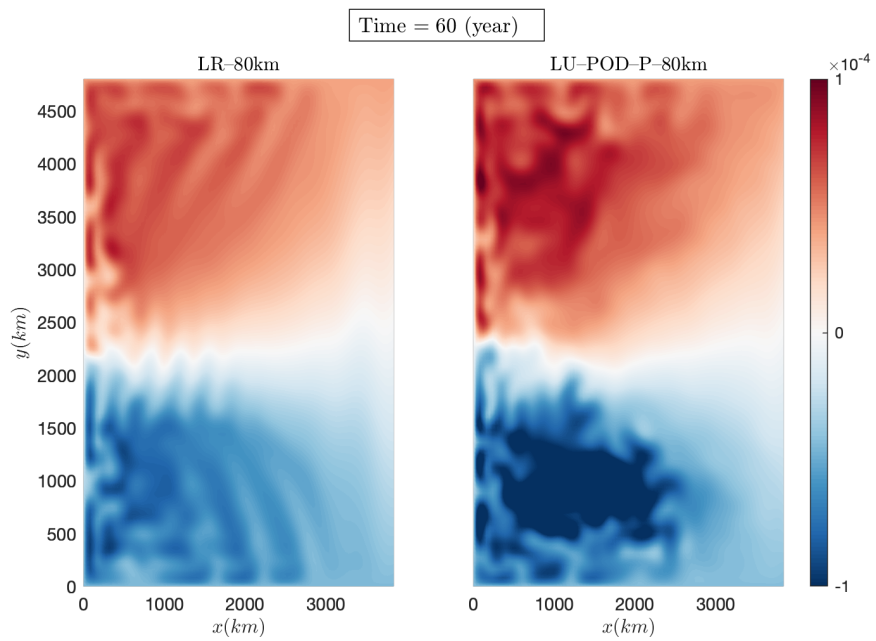

Video: Evolution of surface layer PV at resolution $80 \mathrm{~km}$. Left: Deterministic model (LR);

Right: LU model under POD-P noise. 


\section{LU coarse simulation (Exp.2)}

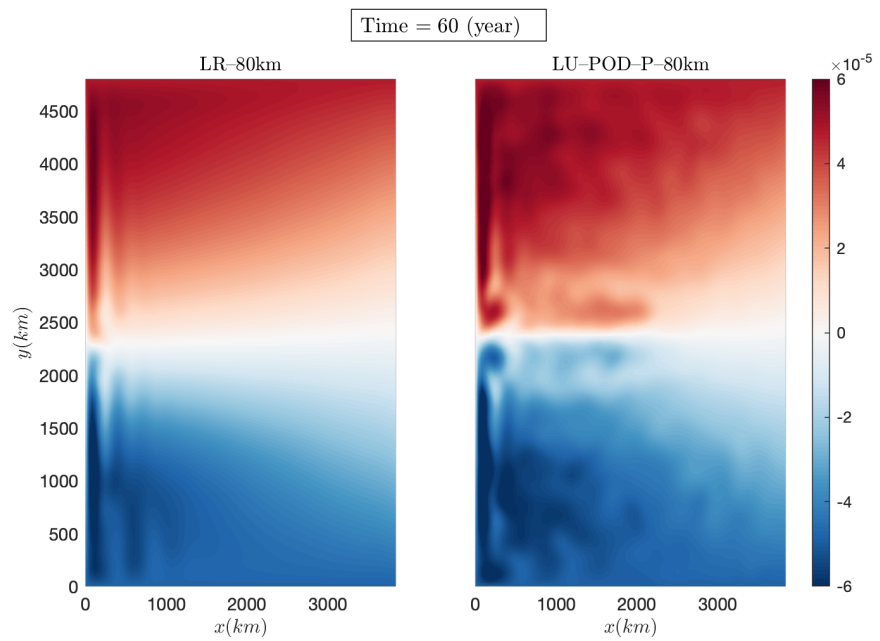

Video: Evolution of surface layer PV at resolution $80 \mathrm{~km}$. Left: Deterministic model (LR);

Right: LU model under POD-P noise. 


\section{LU coarse simulation}

\section{Exp.1}

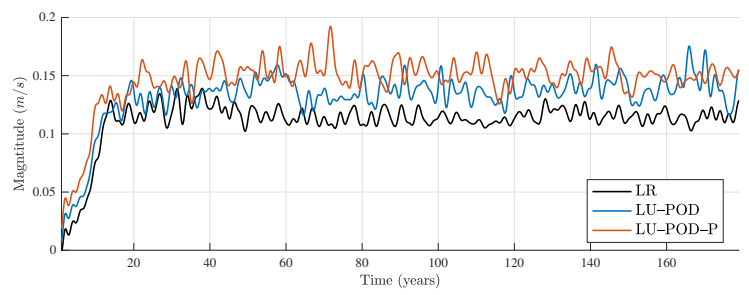

\section{Exp.2}

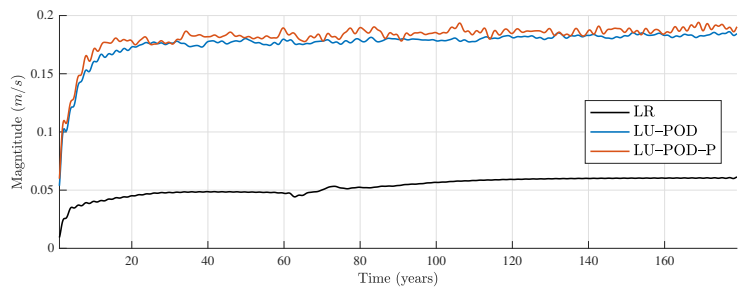

Figure: Maximal values of zonal jet velocity (sum over all layers) at resolution $80 \mathrm{~km}$. 


\section{Low-frequency variability diagnosis}

\section{Eddy kinetic energy (EKE) decomposition}

For $k=1, \ldots, N$ (with $N$ total number of ocean layers):

$$
\mathrm{KE}_{k}=\frac{\rho H_{k}}{2}(\underbrace{\left(\bar{u}_{k}^{2}+\bar{v}_{k}^{2}\right)}_{\text {Standing EKE }}+\underbrace{\left(u_{k}^{\prime 2}+v_{k}^{\prime 2}\right)}_{\text {Transient EKE }}),
$$

where $\cdot$ is approximated by a 2 -years-low-pass Fourier filter and.$'$ is the residual. 


\section{Low-frequency variability diagnosis (Exp.1)}

\section{Standing EKE}

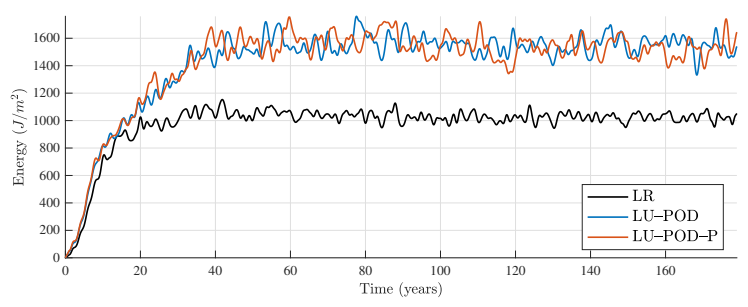

Transient EKE

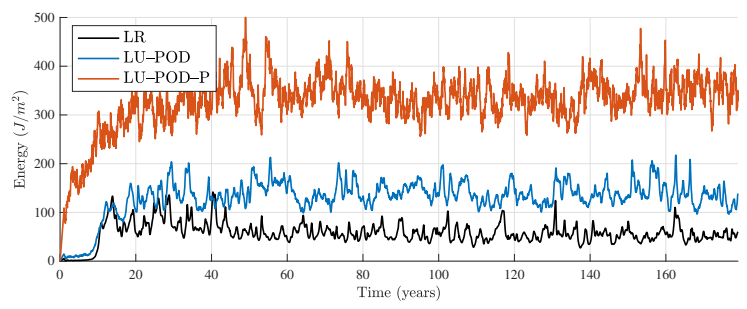

Figure: Standing EKE and Transient EKE (sum over all layers) at resolution $80 \mathrm{~km}$. 


\section{Low-frequency variability diagnosis (Exp.2)}

\section{Standing EKE}

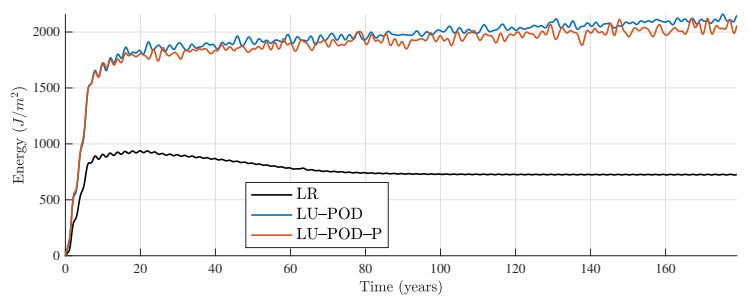

Transient EKE

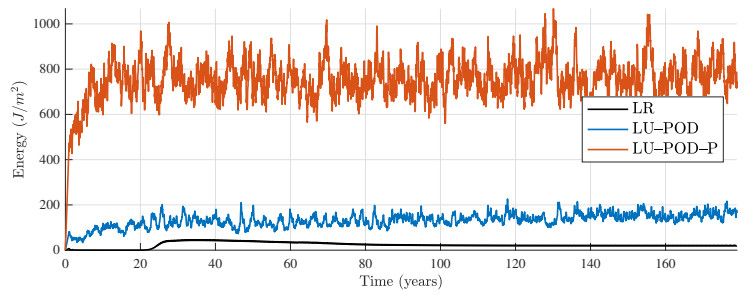

Figure: Standing EKE and Transient EKE (sum over all layers) at resolution $80 \mathrm{~km}$. 


\section{Low-frequency variability diagnosis}

\section{Energy transfert (Hogg and Blundell, 2006)}

$$
\begin{aligned}
\partial_{t} \mathrm{KE}= & \underbrace{-\partial_{t} \mathrm{PE}}_{\text {source }} \underbrace{+\rho \int_{\Omega} u_{1} \tau^{x} \mathrm{~d} \boldsymbol{x}}_{\text {input wind }} \\
& \underbrace{-\frac{1}{2} \rho \delta_{e k} f_{0} \int_{\Omega}\left(u_{N}^{2}+v_{N}^{2}\right) \mathrm{d} \boldsymbol{x}}_{\text {linear drag }} \\
& \underbrace{-\sum_{k=1}^{N} A_{4} \rho H_{k} \int_{\Omega}\left(u_{k} \nabla^{4} u_{k}+v_{k} \nabla^{4} v_{k}\right) \mathrm{d} \boldsymbol{x}}_{\text {dissipation }}+\cdots
\end{aligned}
$$




\section{Low-frequency variability diagnosis (Exp.1)}
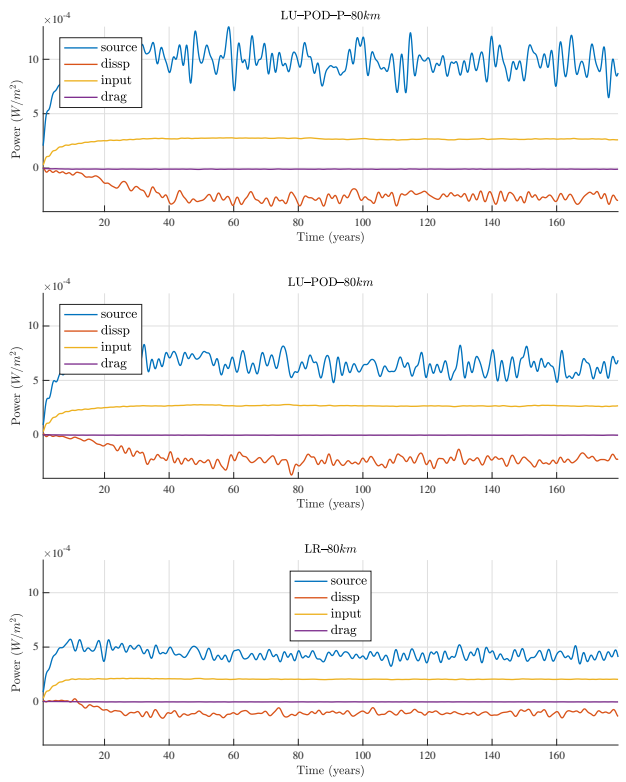

\section{Long Li (Fluminance)}

Stochastic ocean modeling

September 22, 2020

$22 / 29$ 


\section{Low-frequency variability diagnosis (Exp.2)}
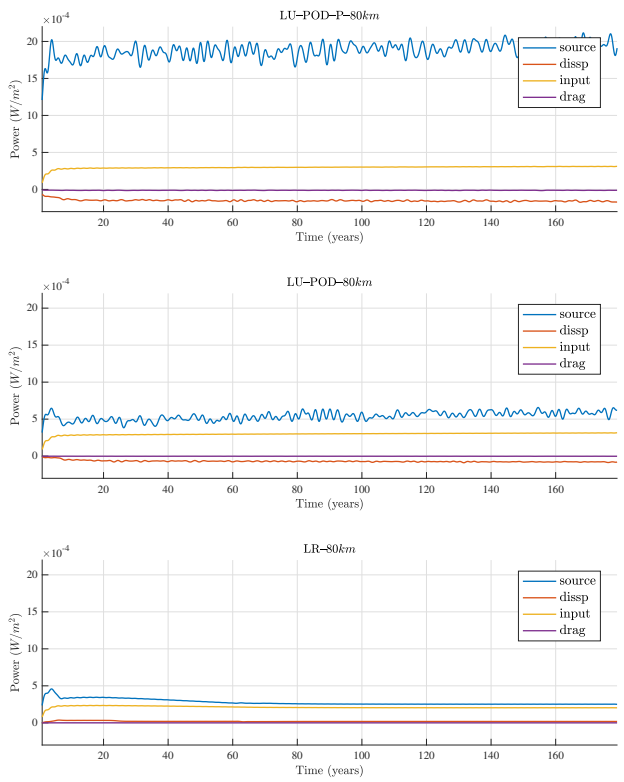

\section{Long Li (Fluminance)}

Stochastic ocean modeling

September 22, 2020

$23 / 29$ 


\section{Low-frequency variability diagnosis}

\section{Measures of statistics (Grooms et al., 2015)}

- Pattern correlation (PC)

$$
\mathrm{PC}=\frac{\int_{\Omega} \sigma_{f}^{2} \sigma_{f_{\text {ref }}}^{2} \mathrm{~d} \boldsymbol{x}}{\left(\int_{\Omega} \sigma_{f}^{4} \mathrm{~d} \boldsymbol{x}\right)\left(\int_{\Omega} \sigma_{f_{\text {ref }}}^{4} \mathrm{~d} \boldsymbol{x}\right)} \quad \nearrow
$$




\section{Low-frequency variability diagnosis}

\section{Measures of statistics (Grooms et al., 2015)}

- Pattern correlation (PC)

$$
\mathrm{PC}=\frac{\int_{\Omega} \sigma_{f}^{2} \sigma_{f_{\text {ref }}}^{2} \mathrm{~d} \boldsymbol{x}}{\left(\int_{\Omega} \sigma_{f}^{4} \mathrm{~d} \boldsymbol{x}\right)\left(\int_{\Omega} \sigma_{f_{\text {ref }}}^{4} \mathrm{~d} \boldsymbol{x}\right)} \quad \nearrow
$$

- Gaussian approximation of relative entropy

$$
\begin{aligned}
& \text { Dispersion }=\frac{1}{|\Omega|} \int_{\Omega}\left(\frac{\sigma_{f_{\text {ref }}}^{2}}{\sigma_{f}^{2}}-1-\log \left(\frac{\sigma_{f_{\text {ref }}}^{2}}{\sigma_{f}^{2}}\right)\right) \mathrm{d} \boldsymbol{x} \searrow \\
& \text { Entropy }=\frac{1}{2}\left(\frac{1}{|\Omega|} \int_{\Omega} \frac{\left(\bar{f}^{t}-\bar{f}_{\text {ref }}^{t}\right)^{2}}{\sigma_{f}^{2}} \mathrm{~d} \boldsymbol{x}+\text { Dispersion }\right) \searrow
\end{aligned}
$$




\section{Low-frequency variability diagnosis (Exp.1)}

\begin{tabular}{cccccc}
\hline Model & RMSE of mean & RMSE of std & PC of std & Dispersion & Entropy \\
\hline LR & 0.44 & 0.71 & 0.44 & 851 & 26 \\
LU-POD & 0.44 & 0.69 & 0.46 & 36 & 18 \\
LU-POD-P & 0.41 & 0.66 & 0.50 & 15 & 8 \\
\hline
\end{tabular}

Table: Measures of skill for surface layer pressure $(80 \mathrm{~km})$.

\begin{tabular}{cccccc}
\hline Model & RMSE of mean & RMSE of std & PC of std & Dispersion & Entropy \\
\hline LR & 0.26 & 0.60 & 0.58 & 188 & 94 \\
LU-POD & 0.26 & 0.59 & 0.61 & 67 & 34 \\
LU-POD-P & 0.25 & 0.55 & 0.70 & 18 & 9 \\
\hline
\end{tabular}

Table: Measures of skill for middle layer pressure $(80 \mathrm{~km})$.

\begin{tabular}{cccccc}
\hline Model & RMSE of mean & RMSE of std & PC of std & Dispersion & Entropy \\
\hline LR & 0.12 & 0.67 & 0.56 & 1701 & 851 \\
LU-POD & 0.12 & 0.65 & 0.65 & 248 & 124 \\
LU-POD-P & 0.12 & 0.59 & 0.80 & 40 & 20 \\
\hline
\end{tabular}

Table: Measures of skill for bottom layer pressure $(80 \mathrm{~km})$. 


\section{Low-frequency variability diagnosis (Exp.2)}

\begin{tabular}{cccccc}
\hline Model & RMSE of mean & RMSE of std & PC of std & Dispersion & Entropy \\
\hline LR & 1.89 & 1.51 & 0.11 & $1 \mathrm{e} 4$ & $5 \mathrm{e} 3$ \\
LU-POD & 1.16 & 1.41 & 0.29 & 156 & 82 \\
LU-POD-P & 1.17 & 1.25 & 0.54 & 19 & 12 \\
\hline
\end{tabular}

Table: Measures of skill for surface layer pressure $(80 \mathrm{~km})$.

\begin{tabular}{cccccc}
\hline Model & RMSE of mean & RMSE of std & PC of std & Dispersion & Entropy \\
\hline LR & 0.47 & 1.16 & 0.26 & $5 \mathrm{e} 4$ & $2 \mathrm{e} 4$ \\
LU-POD & 0.41 & 1.07 & 0.78 & 143 & 72 \\
LU-POD-P & 0.41 & 0.90 & 0.90 & 15 & 8 \\
\hline
\end{tabular}

Table: Measures of skill for middle layer pressure $(80 \mathrm{~km})$.

\begin{tabular}{cccccc}
\hline Model & RMSE of mean & RMSE of std & PC of std & Dispersion & Entropy \\
\hline LR & 0.17 & 1.15 & 0.23 & $2 \mathrm{e} 5$ & $1 \mathrm{e} 5$ \\
LU-POD & 0.16 & 1.06 & 0.93 & 169 & 85 \\
LU-POD-P & 0.16 & 0.89 & 0.92 & 17 & 9 \\
\hline
\end{tabular}

Table: Measures of skill for bottom layer pressure $(80 \mathrm{~km})$. 


\section{Future works}

(1) Run LU ensemble simulations and verify ensemble spread by Rank histogram, CRPS, etc. 


\section{Future works}

(1) Run LU ensemble simulations and verify ensemble spread by Rank histogram, CRPS, etc.

(2) Add noise into steady wind and study the response of ocean variability. 


\section{Future works}

(2) Run LU ensemble simulations and verify ensemble spread by Rank histogram, CRPS, etc.

(2) Add noise into steady wind and study the response of ocean variability.

(3) Implement the Atmosphere-ocean coupled model (Hogg et al., 2003). 


\section{Future works}

(2) Run LU ensemble simulations and verify ensemble spread by Rank histogram, CRPS, etc.

(2) Add noise into steady wind and study the response of ocean variability.

(3) Implement the Atmosphere-ocean coupled model (Hogg et al., 2003).

(4) Data assimilation with particle filter (Cotter et al., 2020). 
Thank for Your Attention! 


\section{References}

W. Bauer, P. Chandramouli, B. Chapron, L. Li, and E. Mémin. Deciphering the role of small-scale inhomogeneity on geophysical flow structuration: a stochastic approach. Journal of Physical Oceanography, 50(4):983-1003, 2020a.

W. Bauer, P. Chandramouli, L. Li, and E. Mémin. Stochastic representation of mesoscale eddy effects in coarse-resolution barotropic models. Ocean Modelling, 151:101646, 2020b.

C. Cotter, D. Crisan, D. D. Holm, W. Pan, and I. Shevchenko. Data assimilation for a quasi-geostrophic model with circulation-preserving stochastic transport noise. Journal of Statiscal Physics, 2020.

P. R. Gent and J. C. McWilliams. Isopycnal mixing in ocean circulation models. Journal of Physical Oceanography, 20(1):150-155, 1990.

I. Grooms, A. J. Majda, and K. S. Smith. Stochastic superparameterization in a quasigeostrophic model of the Antarctic Circumpolar Current. Ocean Modelling, 85:1-15, 2015.

A. McC. Hogg and J. R. Blundell. Interdecadal variability of the southern ocean. Journal of Physical Oceanography, 36:1626-1645, 2006.

A. McC. Hogg, W. K. Dewar, P. D. Killworth, and J. R. Blundell. A quasi-geostrophic coupled model (Q-GCM). Monthly Weather Review, 131(10):2261-2278, 2003.

E. Mémin. Fluid flow dynamics under location uncertainty. Geophysical \& Astrophysical Fluid Dynamics, 108(2): 119-146, 2014.

M. H. Redi. Oceanic isopycnal mixing by coordinate rotation. Journal of Physical Oceanography, 12(10):1154-1158, 1982.

V. Resseguier, E. Mémin, and B. Chapron. Geophysical flows under location uncertainty, part I: Random transport and general models. Geophysical \& Astrophysical Fluid Dynamics, 111(3):149-176, 2017. 